\title{
Nutritional determinants of worldwide diabetes: an econometric study of food markets and diabetes prevalence in 173 countries
}

\author{
Sanjay Basu 1,2,3,*, David Stuckler ${ }^{3,4}$, Martin McKee ${ }^{3}$ and Gauden Galea ${ }^{5}$ \\ 'Department of Medicine, University of California San Francisco, 505 Parnassus Avenue, Room M-987, \\ San Francisco, CA 94131, USA: ${ }^{2}$ Division of General Internal Medicine, San Francisco General Hospital, \\ San Francisco, CA, USA: ${ }^{3}$ Department of Public Health and Policy, London School of Hygiene \& Tropical \\ Medicine, London, UK: ${ }^{4}$ Department of Sociology, Cambridge University, Cambridge, UK: ${ }^{5}$ Division of \\ Noncommunicable Diseases \& Health Promotion, WHO Regional Office for Europe, Copenhagen, Denmark
}

Submitted 17 January 2012: Final revision received 13 April 2012: Accepted 2 May 2012: First published online 13 June 2012

\begin{abstract}
Objective: Ageing and urbanization leading to sedentary lifestyles have been the major explanations proposed for a dramatic rise in diabetes worldwide and have been the variables used to predict future diabetes rates. However, a transition to Western diets has been suggested as an alternative driver. We sought to determine what socio-economic and dietary factors are the most significant population-level contributors to diabetes prevalence rates internationally.

Design: Multivariate regression models were used to study how market sizes of major food products (sugars, cereals, vegetable oils, meats, total joules) corresponded to diabetes prevalence, incorporating lagged and cumulative effects. The underlying social determinants of food market sizes and diabetes prevalence rates were also studied, including ageing, income, urbanization, overweight prevalence and imports of foodstuffs.

Setting: Data were obtained from 173 countries.

Subjects: Population-based survey recipients were the basis for diabetes prevalence and food market data.

Results: We found that increased income tends to increase overall food market size among low- and middle-income countries, but the level of food importation significantly shifts the content of markets such that a greater proportion of available joules is composed of sugar and related sweeteners. Sugar exposure statistically explained why urbanization and income have been correlated with diabetes rates.

Conclusions: Current diabetes projection methods may estimate future diabetes rates poorly if they fail to incorporate the impact of nutritional factors. Imported sugars deserve further investigation as a potential population-level driver of global diabetes.
\end{abstract}

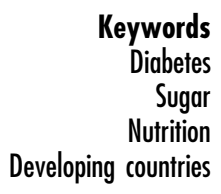

ords

Sugar

Nutrition

Developing countries
Type II diabetes has emerged as a leading cause of death and disability worldwide ${ }^{(1,2)}$. In 2010, an estimated 285 million people were living with diabetes ${ }^{(3)}$. Although often seen as a disease of affluence, almost three out of every four of these people live in low- and middle-income countries, with increasing evidence suggesting that diabetes transitions over time from a disease of affluence to a disease of the poor ${ }^{(4)}$. The number of people with diabetes in developing nations is expected to rise $69 \%$ by the year $2030^{(3)}$ - outpacing even the 'pessimistic scenario' forecasts of earlier global disease models ${ }^{(5)}$.

Why has diabetes risen so rapidly in the developing world, at a much higher rate than that predicted based on our knowledge from high-income countries? According to recent reviews, most efforts to mitigate the problem in developing countries have focused on increasingly sedentary lifestyles contributing to obesity ${ }^{(6-8)}$. However, this begs the question of how to intervene, as at present only very aggressive programmes, such as those implemented in Singapore, have been able to reverse a rise in obesity rates at a population level through exercise-based approaches $^{(9)}$. Despite clinical trials showing that such interventions to reduce obesity could help prevent diabetes and its complications ${ }^{(9,10)}$, the real-world implementation of activity-based efforts has had disappointingly low effectiveness $^{(9)}$.

One recent but contested proposal in high-income countries is to additionally focus on the food environment, 
for example by reducing the intake of high-sugar foods through taxes and related regulations ${ }^{(11,12)}$. Large prospective epidemiological studies demonstrate not only that overall joules contribute to obesity and diabetes, but also that the sugar content of foods is significantly associated with the onset of type II diabetes ${ }^{(13,14)}$. Products with refined sugars confer a high glycaemic load while having poor satiating properties, which is thought to contribute to excessive weight gain, the metabolic syndrome and insulin resistance ${ }^{(15-17)}$. However, emerging data also indicate that refined sugars such as fructose, which are common in processed foods, may contribute to diabetes independently of their impact on obesity, by altering the physiology of insulin responses to induce insulin resistance and impaired glucose tolerance ${ }^{(18-20)}$. While concerns have been raised about sugar-containing food products in Western nations, do such products significantly contribute to diabetes worldwide, such that they should become a point of interest for public health policy makers?

To date, other major hypotheses have been put forward as being the principal determinants of diabetes in developing countries. One commonly held view is that demographic shift, particularly population ageing, is the major driver of diabetes emergence. As the WHO stated, 'age is the single most important determinant' of diabetes $^{(21)}$, based on the increasing prevalence of diabetes with age ${ }^{(22)}$. Urbanization has also been invoked as a second determinant of diabetes ${ }^{(23)}$ : 'rising prevalence of diabetes in India and other developing countries is chiefly attributed to urbanization', reported some researchers, under the premise that city living is conducive to a more sedentary lifestyle than life in rural agricultural zones ${ }^{(24)}$.

Curiously, while ageing and urbanization have been used to estimate the future trajectory of diabetes, the models based on these two factors have notably underestimated actual rates of diabetes now being observed in low- and middle-income countries ${ }^{(5,25)}$. Other social and economic changes besides ageing and urbanization may therefore be contributing increases in diabetes rates ${ }^{(26)}$. A third hypothesis is that food markets integrate into the global economy and as per capita income increases, people shift their dietary habits - a 'nutrition transition' in which people in developing countries spend a greater percentage of their money on processed foods, including new imports ${ }^{(27,28)}$. Unhealthy foods with high sugar, fat and oil content are becoming cheaper even for people who have not experienced a rise in income ${ }^{(29)}$, leading to a phenomenon of 'dietary dependency' in which integration into global markets increases access to, and associated consumption of, foods high in sugar and related carbohydrates $^{(30,31)}$. It is thus plausible that all three factors ageing, urbanization and the nutrition transition - are contributing to the global diabetes pandemic. Indeed, urbanization (due to increased access to unhealthy foods) and economic development (due to rising personal incomes) may be underlying factors contributing to the nutrition transition.

While urbanization, ageing and development are difficult to modify, are particular components of the diet appropriate policy targets for those who wish to stem the rising tide of diabetes? In the current paper we use data available from 173 countries to test the potential role of various nutritional components on the global food market. Our rationale was to use comparable data on diabetes prevalence worldwide and determine what statistical correlates might explain the variation in diabetes rates among countries, when controlling for other intervening factors such as differing levels of economic development. Our study starts with measures of proximal causes of varying diabetes rates - analysing how diabetes prevalence relates to physical inactivity, obesity as well as kilojoule exposure to different types of foods (if they have any effect independent of obesity). We then assess which underlying factors, from urbanization to ageing to economic growth to market integration, can explain significant proximal risks and overall diabetes rates.

\section{Experimental methods}

Data on diabetes prevalence by country (the percentage of adults aged 20 to 79 years with diabetes) was obtained from the International Diabetes Federation's (IDF) Diabetes Atlas Database for the year $2007^{(32)}$. We use the 2007 data because this is the year for which comparative estimates of other key variables of interest (described below) are also available and because the 2007 IDF data have been subject to extensive checks on quality; furthermore, longitudinal comparisons of the IDF data are not recommended due to changes in data collection strategy between years ${ }^{(32)}$.

To assess the population determinants of diabetes, the analysis proceeded in two steps. First, we examined the relationship between diabetes prevalence and proximate risk factors for diabetes. Given the potential importance of nutrition as a risk factor for diabetes, we performed the regression of the prevalence of diabetes $v$. country-specific food marketplace data taken from the FAO describing market size (in kJ/person per d) of: (i) sugars and related sweeteners; (ii) cereals; (iii) fruits and vegetables; (iv) vegetable oils and related fats, (v) meats and other animal products; and (vi) overall total joules ${ }^{(33)}$. The food market size data are considered a standard measure of population exposure to different food items. Of note, 'related sweeteners' refers to carbohydrates like high- fructose corn syrup and honey, which are broken down into blood glucose, not sugar substitute products used as alternatives to sugar (e.g. saccharine or sucralose). Because diabetes develops after cumulative exposure to dietary risks (i.e. high intake of joules today does not lead to immediate obesity, but a prolonged exposure to high joules is required), we calculated 
the mean kilojoules per person per day over a 10-year period in each of these food categories to represent typical long-term exposure to each of these dietary components. The rationale for this decision is that the pathophysiology of diabetes is as a delayed-onset disorder, in which the metabolic syndrome leading from underlying risk factors to frank diabetes mellitus symptomatology is thought to take roughly one decade ${ }^{(34)}$. We also incorporated measures of sedentary lifestyle and obesity as two other proximal determinants of diabetes rates; the number of passenger cars per 1000 people was used as a standard international indicator of sedentary lifestyle (along with urbanization), and the prevalence of both overweight and obese adults (percentage of adults with $\mathrm{BMI} \geq 25 \mathrm{~kg} / \mathrm{m}^{2}$ ) was incorporated as a measure of excess weight in the population.

We also analysed how well key hypothesized population-level risk factors for diabetes - ageing, urbanization, rising incomes and market integration - relate to diabetes prevalence as well as any significant proximal risk correlates to diabetes prevalence. As described further below, we found that sugar exposure was the major proximal dietary risk correlate to diabetes prevalence, independent of obesity or sedentary lifestyle, so we investigated specifically how the underlying population-level factors may relate to sugar exposure as well as to diabetes prevalence. Data on sugar exposure are available longitudinally (not just for the year 2007), so we conducted a full time-series (panel) data analysis for this segment of the study.

Thus, our cross-national statistical models were as follows:

$$
\begin{gathered}
\text { Diabetes }_{i}=\alpha+\beta \text { DIET }_{i}+\beta \mathrm{FAT}_{i}+\beta \mathrm{SED}_{i}+\varepsilon_{i} \\
\text { Diabetes }_{i}=\alpha+\beta \mathrm{SOC}_{i}+\varepsilon_{i} \\
\text { Sugar }_{i, t}=\alpha+\beta \mathrm{SOC}_{i, t}+\mu_{i}+\varepsilon_{i, t}
\end{gathered}
$$

where $i$ is the country, $\alpha$ is the constant in the model, $\beta$ is the individual regression coefficient for each variable, $\mu$ is a country-specific dummy for the fixed-effects time-series regression (correcting for differences inherent to each country between years in the sugar analysis, to avoid confounding by surveillance biases, as judged appropriate by a Hausman test), $t$ is time, and $\varepsilon$ is the error term. DIET is the average market size over 10 years in kilojoules of exposure per person per day for each food category prior to the year 2007 (sugars, labelled SUGAR; cereals; vegetable oils; meats; and total joules); FAT is the percentage of adults who are overweight; SED is the number of passenger cars per 1000 people. In the second stage of models testing population factors leading to dietary change and/or overall diabetes rates, SOC is one of the following social, demographic and economic determinants, testing each underlying hypothesis in turn: (i) AGE, the percentage of the population aged 65 years and above; (ii) URBAN, the percentage of the population living in urban areas as determined by the UN World Urbanization Prospects group (both expressed as percentages to allow comparison with international health impact assessments conducted by the World Bank); (iii) GDP, the measure of average income (per capita, in constant 2005 \$US adjusted for purchasing power parity for cross-country comparability); and (iv) MKT, the number of dollars spent on food imports per capita (in constant 2005 $\$$ US for comparability). We tested each of these SOC variables separately in order to tease apart their individual contributions to diabetes prevalence. All food data were from the FAO's FAOSTAT database from the years 1960 to 2007 (the full duration of data available); all other data were taken from the World Bank World Development Indicators Database 2011 edition and were available for the year 2007 to compare against diabetes prevalence, with the exception for overweight prevalence and passenger cars, for which 2005 data were available ${ }^{(33,35)}$.

\section{Results}

Table 1 shows the results of our models studying the proximal correlates to diabetes prevalence. Model 1 shows that higher total joules were significantly associated with greater diabetes prevalence $(P<0 \cdot 001)$. However, in investigating the dietary components corresponding to diabetes prevalence, specific dietary factors, rather than the overall level of joules, were critically correlated with diabetes. In particular, once sugar and sweeteners were included in the model the effect of total joules disappeared, suggesting that high sugar was the principal determinant of the association between high joules and diabetes. As shown in Table 1, each additional exposure to sugars and related sweeteners of $100 \mathrm{~kJ} /$ person per $\mathrm{d}$ was associated with a $2 \cdot 8 \%$ rise in diabetes prevalence in a country, even after accounting for other components of the diet such as oils and meats $(P<0 \cdot 001)$. The only other dietary factor significantly correlated with diabetes prevalence was cereals $(P<0 \cdot 01)$, which are also high in carbohydrates.

Sedentary lifestyles and overweight were also significant correlates to diabetes prevalence. As shown in Table 1, sugar remained a significant predictor of diabetes independent of these factors $(P<0 \cdot 001)$. Notably, sugar correlated with prevalence of overweight more strongly than did any other component of the diet $(r=0 \cdot 69$; see Supplementary Materials, Part 1).

Figure 1 shows the unadjusted correlation between exposure to sugar and related sweeteners and diabetes prevalence. It shows uneven patterns of sugar exposure worldwide, ranging from $<10 \mathrm{~kJ} /$ person per $\mathrm{d}$ in poorer regions to $>150 \mathrm{~kJ} /$ person per $\mathrm{d}$ in the USA. Notably, high sugar producers, such as Brazil, Jamaica, Dominica, Costa Rica, Cuba, Mexico, Jamaica, Trinidad and Tobago, exhibit high levels of diabetes prevalence. These sugar producers have very high sugar-to-total joule ratios and, as shown in Fig. 1, experience some of the highest rates of diabetes among low- and middle-income countries. 
Additionally, we evaluated commonly proposed distal population-level risk factors for high diabetes rates: ageing, urbanization and economic development. As shown in Table 2, GDP, sedentary lifestyle and urbanization were significantly associated with increased risks of diabetes, but the significant correlations disappeared once sugar exposure was incorporated into the models. This supports the hypothesis that the observed correlation between income, lifestyle and urbanization with diabetes is driven substantially by increasing sugar exposure. Only ageing remained significantly correlated with diabetes prevalence after sugar was taken into account.

\section{Determinants of sugar exposure}

In view of the importance of sugar as a significant correlate of diabetes, we investigated what factors may be driving the magnitude of sugar exposure. Figure 2 depicts the relationship between economic growth (measured by GDP per capita) and exposure to sugar and related sweeteners. As shown, sugar and sweetener exposure tends to rise rapidly in low- and middle-income countries, reaching about 15\% of dietary joules at income of around \$US 10000 per capita; exposure subsequently plateaus. Supplementary Materials, Part 2 shows that, at around \$US 10000 per capita, consumption is close to $750 \mathrm{~kJ} /$ person per $\mathrm{d}$ - about $2 \cdot 5$-fold the recommended maximum sugar consumption level.

Because data on sugar are comparable longitudinally (unlike data on diabetes prevalence), we used longitudinal models to study what factors in addition to GDP may be critically increasing exposure to sugar and related sweeteners over the past several years. Our models control for country 'fixed effects' - that is, they control for differences between countries that are inherent to each country, such as differences in surveillance infrastructure that could otherwise lead to biases in comparison. We did this using a standard econometric technique in which a dummy variable is created for each country and included in the regressions, so that unobserved variables that are specific to each country - e.g. the strength of a country's public health surveillance system - are controlled for implicitly. Table 3 shows the critical determinants of rising sugar exposure, both in terms of overall joules from sugar and in terms of the proportion of sugar in the diet relative to other food (joules of sugar as a percentage of overall joules). Higher GDP was a significant correlate to overall sugar exposure per capita per day $(P<0 \cdot 01)$. Food importation was a significant correlate of both higher sugar joules overall and an increasing dietary composition of sugar (higher proportion of diet made up by sugar; $P<0.05$ ).

\section{Robustness checks}

We replicated our models using independent non-IDF estimates of diabetes prevalence for the year 2008, based on a recent systematic review of health examination surveys and other epidemiological studies of fasting plasma glucose levels and diabetes prevalence ${ }^{(36)}$. Supplementary 

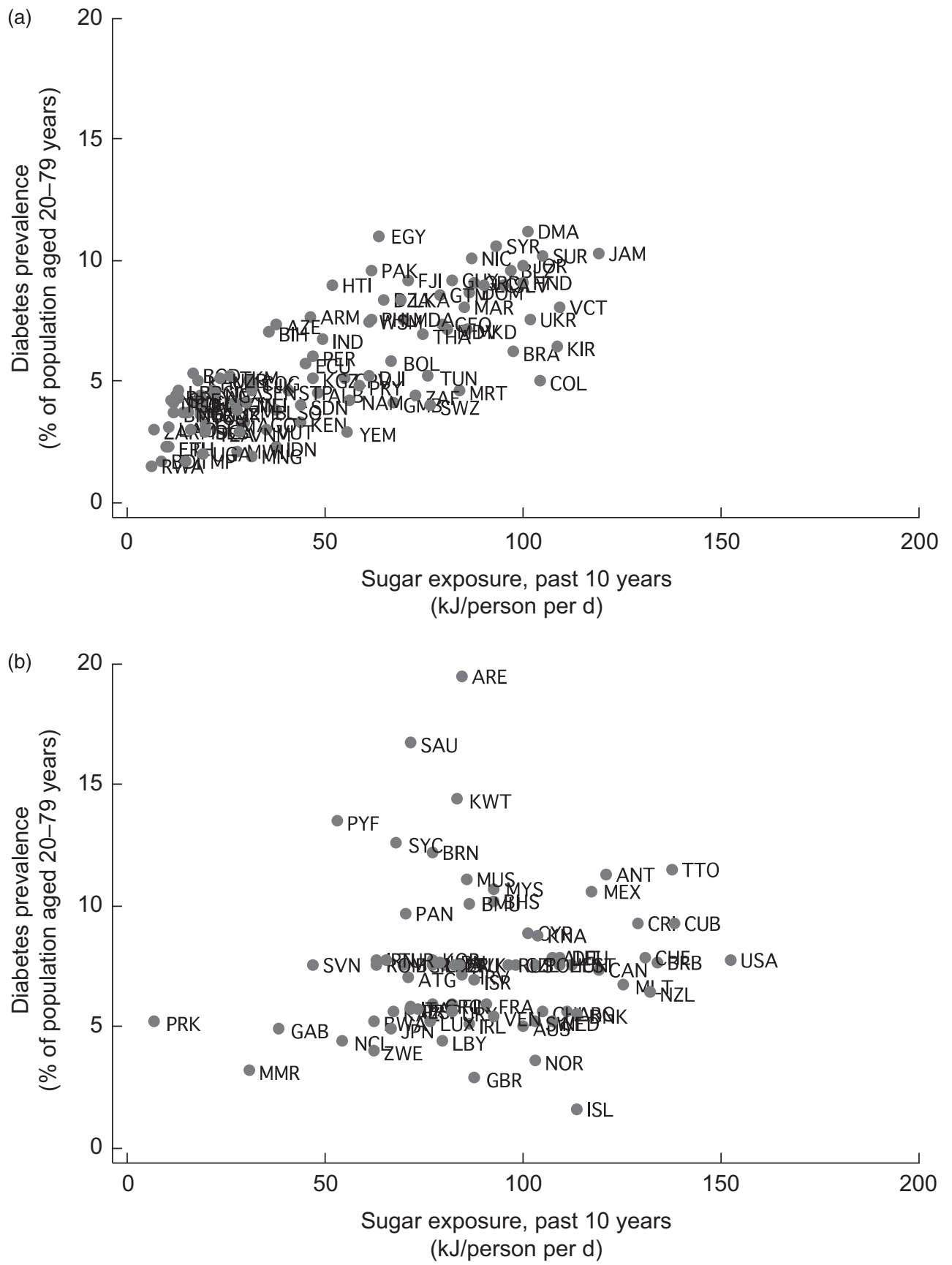

Fig. 1 Sugar exposure and diabetes prevalence, 2007: (a) low- and middle-income countries; (b) high-income countries

Materials, Parts 3 and 4 shows consistency in our results when replicated using the alternative data set.

\section{Discussion}

Our analysis suggests that:

1. The consumption of sugar and related sweeteners, not just total joules, may be a significant determinant of diabetes prevalence.
2. The consumption of sugar and related sweeteners has a statistically significant effect on diabetes that is independent of the effect of weight on diabetes, consistent with emerging data on the insulin-modifying properties of refined sugars.

3. Once the effects of sugar and related sweeteners are taken into account, the correlations between diabetes and both rising incomes and urbanization statistically disappear, indicating that sugar exposure may be an explanation for why urbanization 
Table 2 Underlying determinants of diabetes prevalence, 2007

\begin{tabular}{|c|c|c|c|c|c|c|}
\hline \multirow[b]{3}{*}{ Underlying determinant } & \multicolumn{6}{|c|}{$\%$ Increase in diabetes prevalence with a 1 -unit increase in each factor } \\
\hline & \multicolumn{2}{|c|}{$\begin{array}{l}\text { Without correcting } \\
\text { for impact of sugar }\end{array}$} & \multicolumn{2}{|c|}{$\begin{array}{l}\text { After correcting } \\
\text { for impact of sugar }\end{array}$} & \multicolumn{2}{|c|}{$\begin{array}{l}\text { Impact of sugar when } \\
\text { correcting for each factort }\end{array}$} \\
\hline & $\beta$ & SE & $\beta$ & SE & $\beta$ & SE \\
\hline Percentage of population aged 65 years and above & 0.046 & 0.041 & $-0 \cdot 14^{\star *}$ & $0 \cdot 051$ & $5 \cdot 70^{\star \star *}$ & 0.65 \\
\hline Percentage of population in urban areas & $0.048^{\star \star \star}$ & 0.0090 & 0.0055 & $0 \cdot 012$ & $4 \cdot 40^{\star \star \star}$ & 0.50 \\
\hline Log GDP per capitał & $1 \cdot 12^{* \star *}$ & $0 \cdot 17$ & 0.30 & 0.31 & $3 \cdot 80^{\star * \star}$ & $0 \cdot 87$ \\
\hline Percentage of adults who are overweight & $0.085^{\star \star \star}$ & 0.011 & $0.046^{\star \star}$ & 0.017 & $2 \cdot 90^{\star \star \star}$ & $0 \cdot 70$ \\
\hline $\begin{array}{l}\text { Sedentary lifestyle (number of passenger cars per } \\
1000 \text { people) }\end{array}$ & $0.0035^{\star}$ & 0.0013 & -0.0018 & 0.0021 & $4 \cdot 60^{\star * \star}$ & $0 \cdot 78$ \\
\hline
\end{tabular}

The table describes cross-national models of diabetes prevalence. Results are from twenty separate regression models for each factor's impact on diabetes, estimated in turn with and without the impact of sugar included. Standard errors are robust. $n 173$.

${ }^{\star} P<0.05,{ }^{\star \star} P<0.01,{ }^{\star \star \star} P<0.001$.

tThis is the independent effect of increasing sugar exposure by $100 \mathrm{~kJ} /$ person per $\mathrm{d}$ when each individual factor is included in the model, e.g. Diabetes $_{i}=\alpha+\beta$ SUGAR $_{i}+\beta \mathrm{SOC}_{i}+\varepsilon_{i}$ (see Experimental methods).

¥Gross domestic product in constant 2005 \$US, adjusted for purchasing power parity.

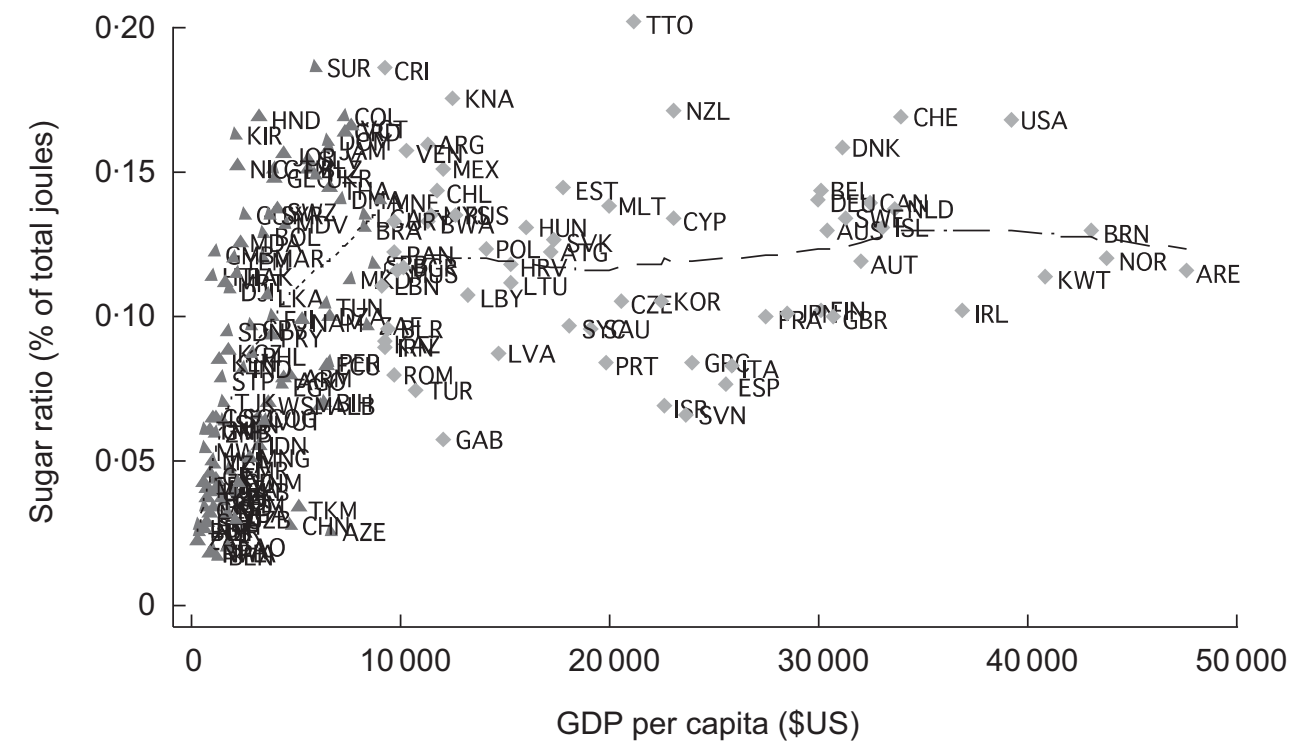

Fig. 2 Sugar exposure and economic development, 2007: $\Delta$, low- and middle-income countries; $\diamond$, high-income countries (GDP, gross domestic product in constant 2005 \$US, adjusted for purchasing power parity)

and rising incomes have been correlated with diabetes rates.

4. Increased income correlates to increased overall joules of food available among low- and middle-income countries (potentially as people move out of poverty and increase food demand). The level of food importation, however, appears to shift the food supply significantly such that a higher proportion of the available dietary joules is composed of sugar and related sweeteners.

Before evaluating the public health implications of our work, it is necessary to note our study's important limitations. First, we must highlight the limitations of the ecological approach that we adopted here. It is neither ethical nor logistically feasible to randomize communities to different levels of poverty, urbanization, ageing or nutritional access to determine which community would experience higher diabetes rates. Hence, we used an observational set of data, which are inherently subject to ecological bias, i.e. 'correlation is not causation'. To minimize the risk of erroneous associations, we adopted a number of control variables as described, but this does not eliminate the potential to correlate variables merely due to their coexistence rather than a true causal relationship. No individual-level relationships could be traced here, but future studies may be able to isolate the association between sugar and diabetes by conducting carefully controlled studies of dietary sugar exposure and subsequent diabetes incidence. Future studies should also conduct longitudinal time-series analysis of the sugar-diabetes relationship once comparable diabetes prevalence data become available. 
We used a standard proxy for our measure of sedentary behaviour, in terms of passenger cars per 1000 people, which was statistically insignificant and may be a poor proxy for such behaviour. However, our inclusion of the prevalence of overweight adults in our models likely captured many of the physiological consequences of sedentary lifestyles. Second, the measure of food imports available was dollars spent on food imports per capita, rather than a more fine-grained measure of food imports as a fraction of total food consumption. It may therefore capture import reliance, but not necessarily proportional dietary changes. Third, our study was unable to identify the specific products driving rising sugar consumption. While we used measures of food market size, as direct measures of how many joules are actually consumed per person are unavailable, our study can be interpreted as reflecting the marketplace of food. The difference between potential consumption and actual consumption is composed of by wastage or smuggling, which are diminished by market competition in the food industry and considered slim in developing countries ${ }^{(33,35)}$.

A final caveat is that all diabetes prevalence data are estimates of a broader population that cannot be entirely sampled. The IDF data contain estimates of national diabetes prevalence derived from heterogeneous survey studies. The data include people with type I diabetes (although 95\% are believed be type II), but we would expect this confounder to produce regression towards the mean, making our results conservative. Also, the diabetes data for some countries are limited; many of sub-Saharan Africa's data are projections from nearby nations and populations, as are data from some former Soviet states and the Caribbean ${ }^{(37)}$. Hence, we also replicated our data with independent data from investigators who estimated diabetes prevalence from a variety of direct epidemiological surveys, attempting to convert systematically the different metrics used ${ }^{(36)}$. The disadvantage of the latter data is that they involve significant mathematical smoothing and are subject to wide uncertainty intervals. Therefore, we also replicated our results using the subset of that study for which direct population data were available (not extrapolations, see Supplementary Materials, Parts $3 \mathrm{~b}$ and $4 \mathrm{~b}$ ), and our results remained robust.

The findings here nevertheless indicate a need to revise models used to estimate future diabetes rates, as these are currently based on rates of ageing and urbanization; newer projections should incorporate dietary risk factors and food imports. Our findings also suggest that the nutrition transition in developing countries may be better understood by analysing the economic forces that underpin diabetes, particularly food markets ${ }^{(38,39)}$. For policy makers, our findings suggest that supply-side food policy measures to address rising exposure to sugars and related sweeteners may be investigated as a strategy to curtail the rise of diabetes in developing nations. 


\section{Acknowledgements}

This research received no specific grant from any funding agency in the public, commercial or not-for-profit sectors. There are no conflicts of interest. S.B. and D.S. conceived of the study, carried out the analysis and interpreted the results. M.M. and G.G. assisted in interpretation of results. All authors contributed to writing of the manuscript.

\section{Supplementary Materials}

For Supplementary Materials for this article, please visit http://dx.doi.org/10.1017/S136898001200288

\section{References}

1. World Health Organization (2009) Mortality and Burden of Disease Estimates for WHO Member States in 2004. Geneva: WHO.

2. King H \& Rewers M (1993) Global estimates for prevalence of diabetes mellitus and impaired glucose tolerance in adults. WHO Ad Hoc Diabetes Reporting Group. Diabetes Care 16, 157-177.

3. Shaw J, Sicree RA \& Zimmet PZ (2010) Global estimates of the prevalence of diabetes for 2010 and 2030. Diabetes Res Clin Pract 87, 4-14.

4. Sicree R, Shaw J \& Zimme P (2010) Diabetes and Impaired Glucose Tolerance. Geneva: IDF.

5. Murray C \& Lopez AD (1996) Global Burden of Disease: A Comprehensive Assessment of Mortality and Disability from Diseases, Injuries and Risk Factors in 1990 and Projected to 2020. Cambridge, MA: Harvard School of Public Health.

6. Prentice AM (2006) The emerging epidemic of obesity in developing countries. Int J Epidemiol 35, 93-99.

7. Hossain P, Kawar B \& El Nahas M (2007) Obesity and diabetes in the developing world - a growing challenge. $N$ Engl J Med 356, 213-215.

8. Seidell JC (2000) Obesity, insulin resistance and diabetes a worldwide epidemic. BrJ Nutr $\mathbf{8 3}$, Suppl. 1, S5-S8.

9. Simmons R, Unwin N \& Griffin S (2010) An Update of the Evidence Concerning the Prevention of Type 2 Diabetes. Geneva: IDF.

10. Ramachandran A, Snehalatha C, Mary S et al. (1997) The Indian diabetes prevention programme shows that lifestyle modification and metformin prevent type 2 diabetes in Asian Indian subjects with impaired glucose tolerance (IDPP-1). Diabetologia 49, 289-297.

11. Brownell K, Farley T, Willett WC et al. (2009) The public health and economic benefits of taxing sugar-sweetened beverages. N Engl J Med 361, 1599-1605.

12. Brownell K \& Frieden TR (2009) Ounces of prevention the public policy case for taxes on sugared beverages. $N$ Engl J Med 360, 1805-1808.

13. Salmeron J, Manson JE, Stampfer MJ et al. (1997) Dietary fiber, glycemic load, and risk of non-insulin-dependent diabetes mellitus in women. JAMA 277, 472-477.

14. Salmeron J, Ascherio A, Rimm EB et al. (1997) Dietary fiber, glycemic load, and risk of NIDDM in men. Diabetes Care 20, 545-550.

15. Ludwig DS (2002) The glycemic index: physiological mechanisms relating to obesity, diabetes, and cardiovascular disease. JAMA 287, 2414-2423.

16. Mourao DM, Bressan J, Campbell WW et al. (2007) Effects of food form on appetite and energy intake in lean and obese young adults. Int J Obes (Lond) 31, 1688-1695.
17. Schulze MB, Manson JE, Ludwig DS et al. (2004) Sugarsweetened beverages, weight gain, and incidence of type 2 diabetes in young and middle-aged women. JAMA 292, 927-934.

18. Elliott SS, Keim NL, Stern JS et al. (2002) Fructose, weight gain, and the insulin resistance syndrome. Am J Clin Nutr 76, 911-922.

19. Bray GA (2007) How bad is fructose? Am J Clin Nutr 86 , 895-896.

20. Yoshida M, McKeown NM, Rogers G et al. (2007) Surrogate markers of insulin resistance are associated with consumption of sugar-sweetened drinks and fruit juice in middle and older-aged adults. $J$ Nutr 137, 2121-2127.

21. Roglic G \& Unwin N (2005) Global mortality attributable to diabetes: time for a realistic estimates. Diabetes Voice 50, 33-34.

22. Yang W, Lu J, Jia W et al. (2010) Prevalence of diabetes among men and women in China. N Engl J Med 362, 1090-1101.

23. Diamond J (2011) Medicine: diabetes in India. Nature 469 , 478-479.

24. Ramachandran A, Mary S, Yamuna A et al. (2008) High prevalence of diabetes and cardiovascular risk factors associated with urbanization in India. Diabetes Care 31, 893-898.

25. King H, Aubert RE \& Herman WM (1998) Global burden of diabetes, 1995-2025: prevalence, numerical estimates, and projections. Diabetes Care 21, 1414-1431.

26. Yusuf S, Reddy S, Ounpuu S et al. (2001) Global burden of cardiovascular diseases: part I: general considerations, the epidemiologic transition, risk factors, and impact of urbanization. Circulation 104, 2746-2753.

27. Drewnowski A \& Popkin BM (1997) The nutrition transition: new trends in the global diet. Nutr Rev 55, 31-43.

28. Reddy KS \& Yusuf S (1998) Emerging epidemic of cardiovascular disease in developing countries. Circulation 97, 596-601.

29. Monteiro CA, Moura EC, Conde WL et al. (2004) Socioeconomic status and obesity in adult populations of developing countries: a review. Bull World Health Organ 82, 940-946.

30. Brownell K \& Warner KE (2009) The perils of ignoring history: Big Tobacco played dirty and millions died. How similar is Big Food? Milbank Q 87, 259-294.

31. Stuckler D (2008) Population causes and consequences of leading chronic diseases: a comparative analysis of prevailing explanations. Milbank $Q$ 86, 273-326.

32. International Diabetes Federation (2011) International Diabetes Federation Diabetes Atlas. http://www.idf.org/ diabetesatlas/ (accessed October 2011).

33. Food and Agriculture Organization of the United Nations (2011) FAOSTAT Statistical Database. Rome: FAO.

34. Fauci AS (2008) Diabetes mellitus. In Harrison's Principles of Internal Medicine, Chapter 344. New York: McGraw-Hill.

35. World Bank (2011) World Development Indicators Database. Washington, DC: IBRD.

36. Danaei G, Finucane MM, Lu Y et al. (2011) National, regional, and global trends in fasting plasma glucose and diabetes prevalence since 1980: systematic analysis of health examination surveys and epidemiological studies with 370 country-years and 2.7 million participants. Lancet 378, 31- 40 .

37. Zhang P, Zhang X, Brown JB et al. (2010) Economic Impact of Diabetes. Geneva: IDF.

38. Popkin B (2002) What is unique about the experience in lower- and middle-income less-industrialised countries compared with the very-high income countries? The shift in the stages of the nutrition transition differs from past experiences! Public Health Nutr 5, 205-214.

39. Reddy KS (2002) Cardiovascular diseases in the developing countries: dimensions, determinants, dynamics and directions for public health action. Public Health Nutr 5, 231-237. 